\title{
LOCALIZED AMYLOID INFILTRATIONS OF THE LOWER RESPIRATORY TRACT
}

\author{
BY
}

\author{
F. WHITWELL
}

\author{
From the Departments of Pathology, Broadgreen and Aintree Hospitals, Liverpool
}

\author{
(RECEIVED FOR PUBLICATION DECEMBER 19, 1952)
}

Amyloid infiltration of organs occasionally occurs without any associated primary or secondary amyloidosis. Such localized deposits frequently produce nodular thickenings of the affected tissues and they are often called amyloid tumours. The commonest sites of amyloid tumours are the respiratory tract, the skin, and the conjunctivae; less frequently they have been described in other tissues, such as the urinary tract, the stomach, muscle, and bone.

In cases affecting the respiratory tract the lesions have been found, in order of frequency, in the larynx, trachea, and pharynx (Figi, 1942). Amyloid deposits of the lower respiratory tract appear to be much rarer, only 10 cases having been found in the literature. Eight of these were discovered at post-mortem examinations, and in the other two cases the diagnosis was made from pneumonectomy specimens which had been removed for suspected bronchial carcinomas.

The present account consists of the description of a case where the diagnosis has been made from bronchial biopsy, with a brief review of earlier cases and a discussion of the condition.

\section{CASE RePORT}

A 48-year-old general labourer at a steel-works, who had always suffered from a slight cough though otherwise enjoying good health, had a small haemoptysis on February 18, 1952. One week later he was examined by Dr. A. C. C. Hughes, who found no abnormal physical signs, though a radiograph showed collapse of the apex of the right lower lobe. On bronchoscopy a granulomatous polyp was found at the lower end of the trachea on the anterior surface, and below this lesion the bronchial tree appeared normal except for a similar mass in the wall of the right lower lobe bronchus, blocking the apical branch. Biopsies taken from both these lesions were reported as showing changes of an inflammatory nature with considerable fibrosis. The patient was given a threeweeks' course of penicillin, and a radiograph taken after his discharge from hospital showed that the right lower lobe had re-aerated. Dr. Hughes' tentative diagnosis at that time was bronchial carcinoma or an ulcerating tuberculous lymph-node.
The patient was referred to Mr. Leslie Temple, who carried out a bronchoscopy at Broadgreen Hospital on May 12. Mr. Temple has made the following report on the bronchoscopic appearances.

"The upper part of the trachea was normal. On the anterior and lateral walls of the lower trachea the mucosa was lax and redundant, and continuous with an almost polypoid irregularity of the mucosa in the anterior part of the carina. This thickening extended into a fold in the left main bronchus, but the remainder of the left bronchial tree was normal. The right main bronchus was narrowed by similar folds in its anterior and posterior walls, and these folds were easily pushed aside by the advancing bronchoscope, transmitting an almost gritty feeling which suggested a rather cornified epithelium. Further down the right bronchial tree the appearance was normal, except for a discrete hard tumour, about $5 \mathrm{~mm}$. diameter, which was situated in the lower lobe bronchus at the origin of the apical branch. The appearance of this tumour suggested a chondroma. Biopsy specimens were taken from this tumour and from the hypertrophic folds at the carina."

Histology.-Two biopsy specimens were examined microscopically.

Right Lower Lobe Snippet.-The bronchial epithelium is intact and composed of columnar cells, beneath which is a thin layer of fibrous connective tissue containing small blood vessels and foci of chronic inflammatory cells. The supporting bronchial tissues are disorganized and extensively destroyed by a homogeneous acellular substance which thickens the bronchial wall.

In sections stained with haematoxylin and eosin this substance has a pale pink colour, and it occurs both as large clumps separated into fragments by a fine fibrous network and as sharply demarcated broad rings which encircle the mucous glands and their ducts. Some of these ducts are constricted, others are dilated and contain inspissated mucin. Some mucous glands are normal, but many are atrophic. Fragments of cartilage are encased in this amorphous material, which does not surround the blood vessels (Fig. 1).

The infiltrating substance stains a khaki colour with Van Gieson stain in sharp contrast to the yellow and red of the muscle and fibrous tissue (Fig. 2), and 
metachromatically with methyl green, crystal green, methyl violet, and toluidine blue. It does not give any characteristic staining reactions with iodine or iodine green, and with periodic acid Schiff stain only the inspissated mucin in obstructed ducts stains prominently. Silver stains show that no reticulin fibres penetrate the substance, though they are condensed around it (Fig. 3). The congo red reaction is strongly positive (Fig. 4), and in these stained sections the stained material is doubly refractile.

Carinal Snippet.-The epithelium is replaced by a rather atrophic layer of squamous cells, beneath which is a thin layer of loose fibrous tissue. The deeper structures consist of denser fibrous tissue interspersed with large clumps of acellular material (Fig. 5 ), which is similar in morphology and staining reactions to the substance described in the lower lobe snippet.

There are only a few traces of mucous glands or cartilage, and the appearance suggests a more superficial biopsy.

On the histological findings the diagnosis was of localized bronchial amyloid infiltrations.

At a later date the original biopsy sections were re-examined, and they were found to contain similar lesions. Further clinical examination showed that the patient had no hepatomegaly or splenomegaly, and no abnormalities were found in blood or urine.

It was decided that no drastic surgical treatment would be of any benefit to this patient, and he was discharged home. He resumed hard manual work, and on examination five months later no clinical or radiological abnormalities were found, and the patient felt perfectly fit.

\section{REVIEW}

Ten cases of amyloid tumours of the lower respiratory tract have been traced in the literature, but it is possible that others have been overlooked. In conformity with previous accounts, lower respiratory tract is taken to include all structures below the bifurcation of the trachea. The more important findings in the recorded cases are summarized in Table $I$; certain features require elaboration.

AgE AND SEX.-The youngest recorded patient was a man of 35 years of age (Case 9), but for reasons which will be discussed later the diagnosis of this case cannot be accepted. The next youngest patient was 48 years old, but seven out of the 11 patients were in their sixth and seventh decades.

Eight of the patients were men. This age and sex incidence shows that the disease has to be differentiated from bronchial carcinoma.

SyMPTOMS.-In four accounts there was no mention of symptoms referable to the respiratory tract. Four patients suffered from dyspnoea, and two patients complained of hoarseness (in these two cases the upper respiratory tract was also affected). Recent haemoptysis was the presenting symptom in one case. Two patients complained of persistent cough, and one of asthma.

The duration of symptoms was extremely variable, ranging from a few weeks to 40 years.

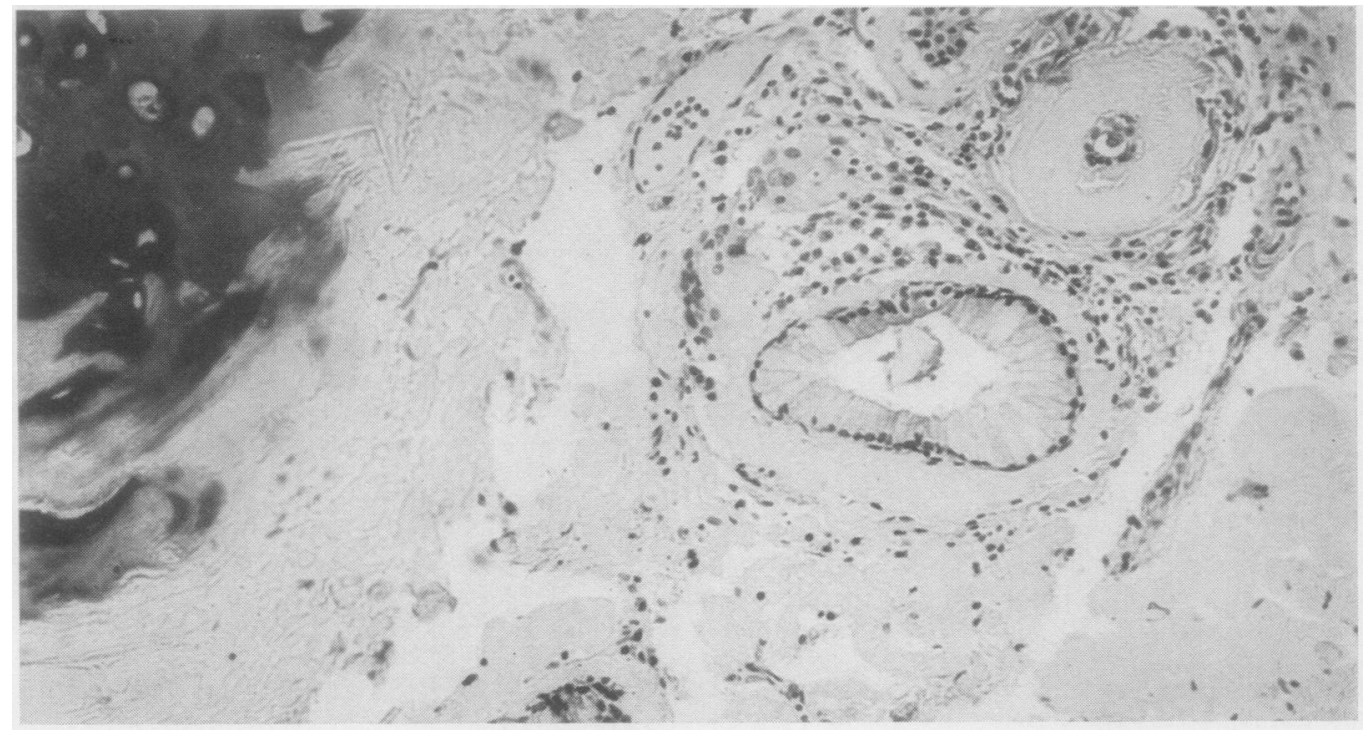

Fig. 1.-Mucous glands and their ducts encircled by amyloid. Connective tissues replaced by amyloid, which erodes the cartilage. Haematoxylin and eosin, $\times 170$. 
Fig. 2.-Inspissated mucin in obstructed dilated mucous gland ducts, which are encircled by amyloid. Iron haematoxylin and van Gieson stain, $\times 170$

Fig. 3.-Reticulin fibres form a fine network in the diffuse amyloid, but no reticulin fibres penetrate the amyloid around the mucous glands and their ducts. Gordon and Sweet's silver stain, $\times 230$.
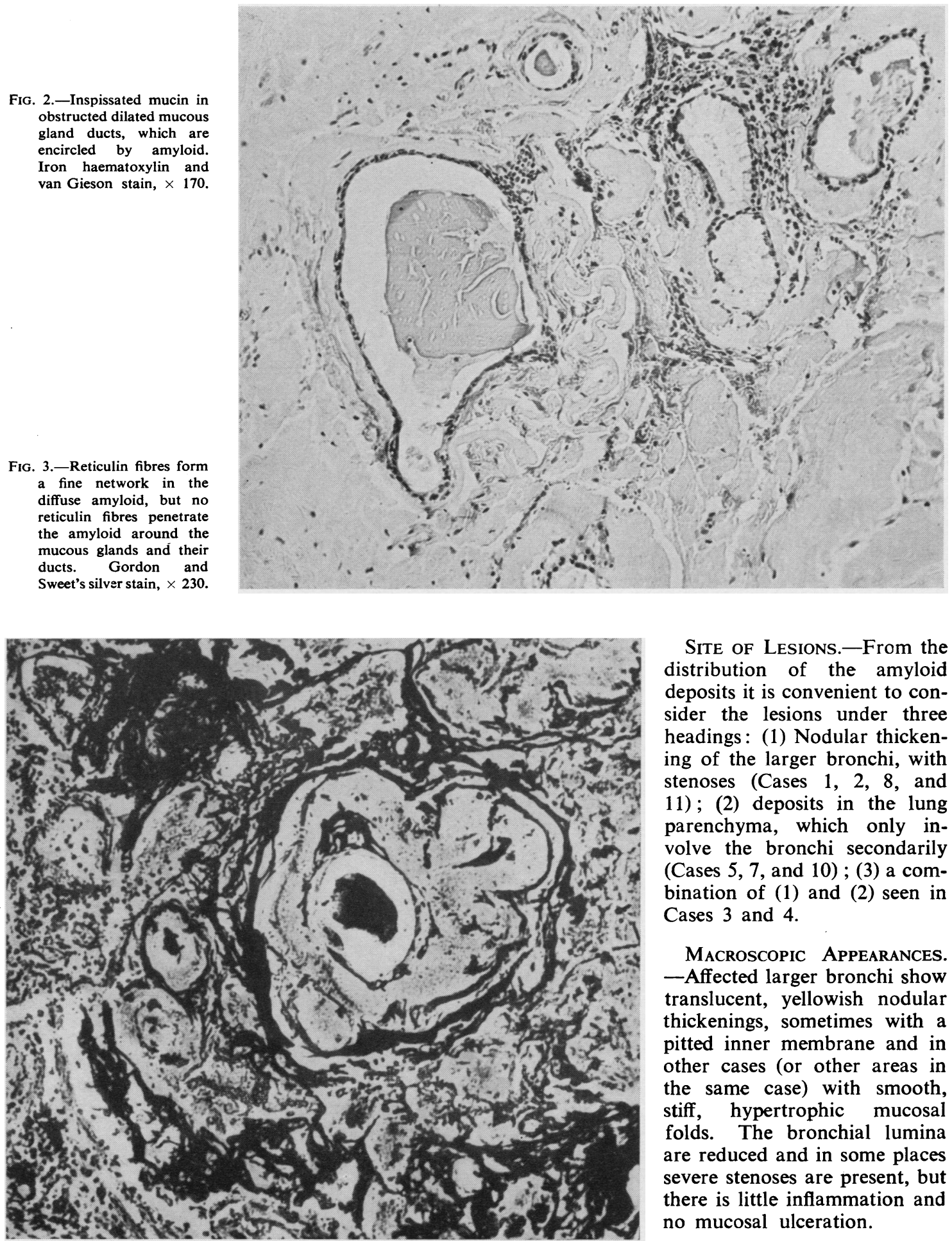

Site of Lesions.-From the distribution of the amyloid deposits it is convenient to consider the lesions under three headings: (1) Nodular thickening of the larger bronchi, with stenoses (Cases 1, 2, 8, and 11); (2) deposits in the lung parenchyma, which only involve the bronchi secondarily (Cases 5, 7, and 10) ; (3) a combination of (1) and (2) seen in Cases 3 and 4 .

Macroscopic Appearances. -Affected larger bronchi show translucent, yellowish nodular thickenings, sometimes with a pitted inner membrane and in other cases (or other areas in the same case) with smooth, stiff, hypertrophic mucosal folds. The bronchial lumina are reduced and in some places severe stenoses are present, but there is little inflammation and no mucosal ulceration. 


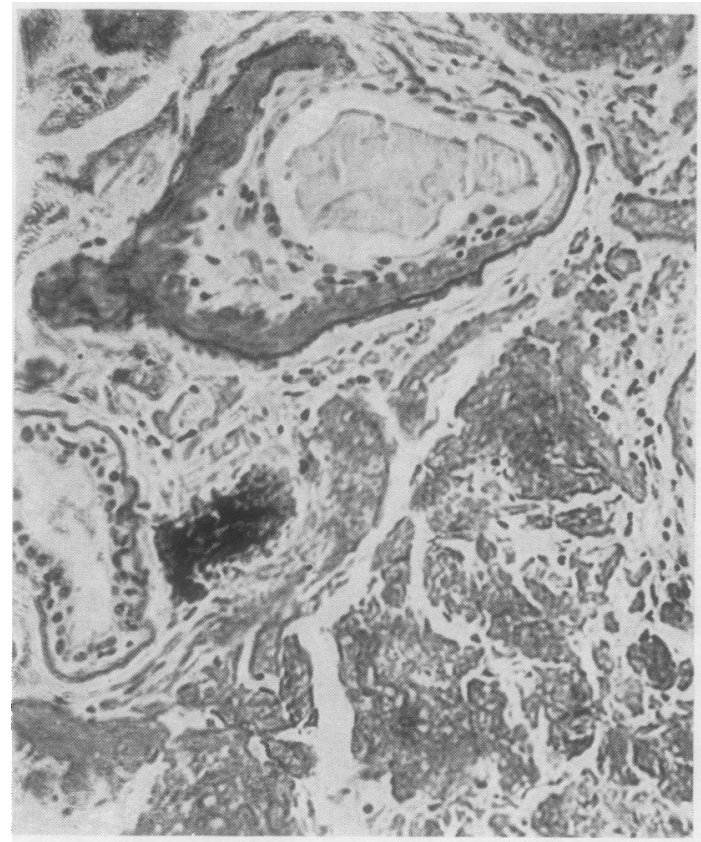

Fig. 4.-Similar lesions to those -in Fig. 3 around mucous glands. Congo red, $\times 190$.

Contiguous structures are often affected, such as the trachea with bronchi, and the larynx with the trachea. This feature was noted by Pollak (1914) as a characteristic of all amyloid tumours. Regional lymph nodes are not involved.

Pulmonary lesions have been described both as wedge-shaped areas with their bases on the pleural membrane (like infarcts), and as rounded masses. They are frequently multiple and bilateral, and vary in size between "a hen's egg" and " a pea." These tumours are firm, and often show a greyyellowish translucence. When perihilar parenchyma is affected there may be some involvement of the bronchi.

Histology OF THE Bronchial Lesions.Characteristic features are: (1) Subepithelial infiltration of an amorphous acellular substance: the overlying epithelium is intact, but may show squamous metaplasia (Fig. 5). (2) Mucous glands and their ducts are encased in thick layers of the same amorphous substance. In section this appears as sharply demarcated laminated broad bands encircling and often constricting the mucous glands and their ducts, with secondary changes in these structures (Figs. 1, 2, and 3). (3) Remains of eroded bronchial cartilages are embedded in the amorphous material (Fig. 1). (4) The staining reactions of this amorphous material are charac-

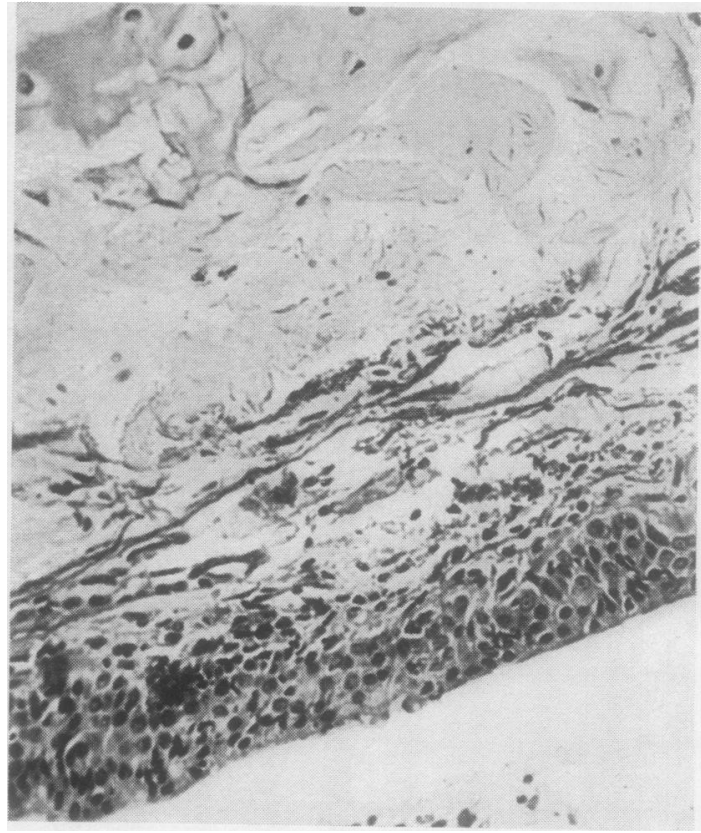

FIG. 5.-Amyloid in subepithelial tissues with epithelial squamous metaplasia. Iron haematoxylin and van Gieson stain, $\times 230$.

teristic of amyloid; i.e., specific staining with iodine and/or the polychromatic stains. Congo red has not been mentioned as a stain in the earlier cases, but in the present case it gave a strongly positive reaction, and the stained amyloid was doubly refractile. (5) In some cases there are giant cells and small foci of calcification among and at the edges of the amyloid.

Histology of THE Lung Lesions.-In the centre of the lesions there is a complete loss of the original structures with rep'acement by amyloid. Near the periphery are zones of transition to the normal surrounding tissues, where it can be seen that the amyloid is arising in the interstitial tissues of the alveolar walls. In some cases the amyloid has been described as arising in the walls of the small arteries, a feature not noted in the bronchial lesions.

The pulmonary amyloid gives the typical staining reactions.

Prognosis.- - Hitherto there has been no chance of studying the natural history of this condition, but the present case will provide an opportunity. More is known about the behaviour of upper respiratory tract lesions, which probably are very similar. According to Kramer and Som (1935) recurrence is common even after wide extirpation, 


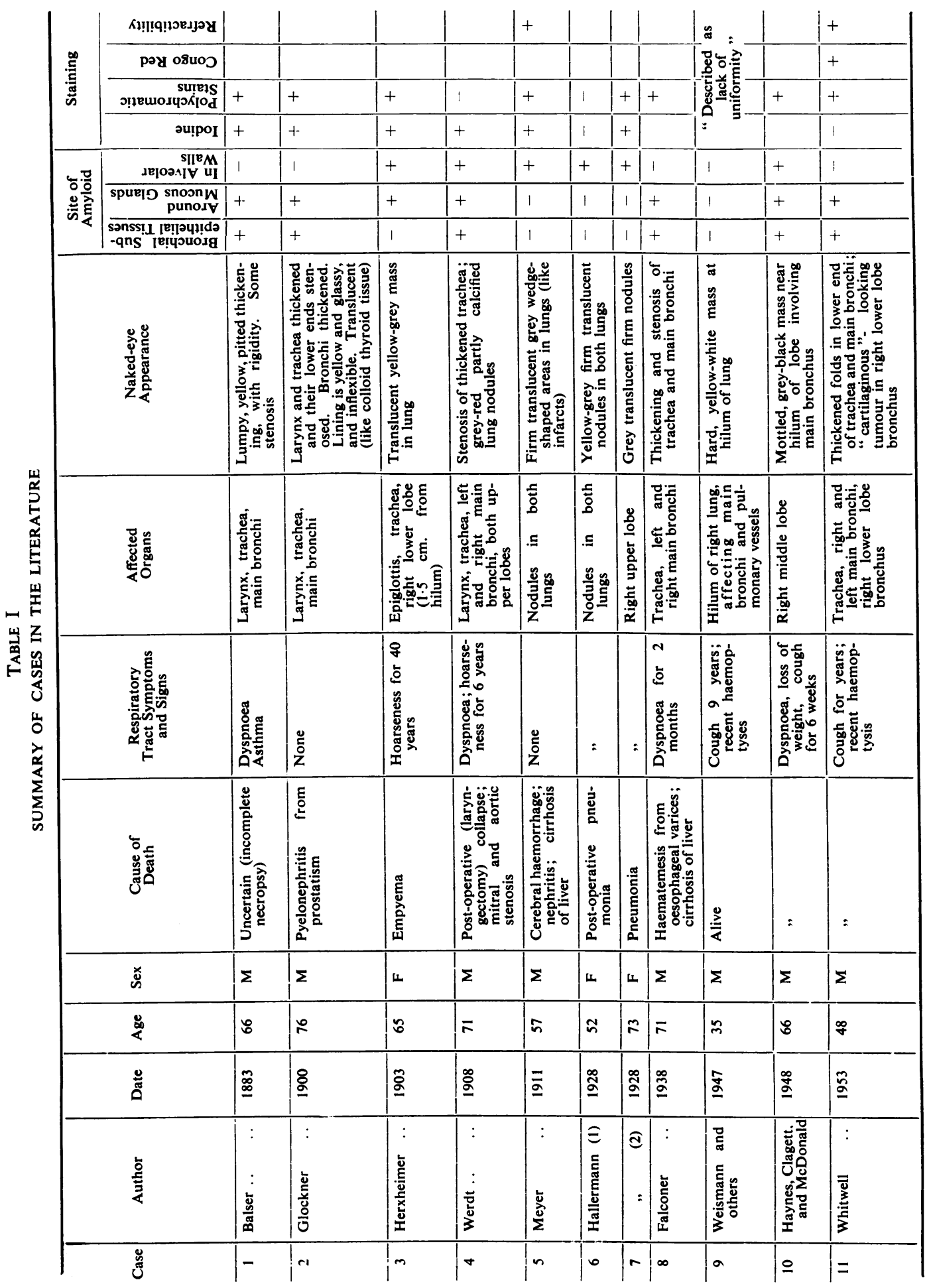

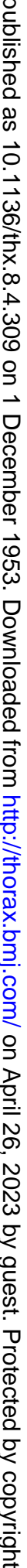


though cases have been described where lesions have regressed after biopsy.

No account has been found of generalized amyloidosis developing as a sequel to local amyloid deposits. Beckert (1917) recorded a case of purpura where an amyloid tumour of the stomach was found at necropsy, but this association was probably fortuitous.

TREATMENT.-No deliberate treatment of lower respiratory tract amyloid deposits has been recorded.

Upper respiratory tract amyloid tumours are usually treated by wide excision, the aim being to prevent respiratory obstruction (New and Erich, 1938). According to Figi (1942), the standard treatment at the Mayo Clinic has been destruction of prominent parts of the lesions with diathermy, followed by repeated irradiation for months; but results have been indifferent.

Weismann, Clagett, and McDonald (1947) consider that amyloid tumours are similar to primary amyloidosis, which they describe as a fatal disturbance from which no recovery has been recorded. For this reason they say "surgical excision or fulguration must be the treatment of choice in the eradication of localized amyloidosis."

Probably the treatment of lower respiratory tract amyloid tumours should depend upon the type of lesions. Bronchial deposits should be treated by endoscopic resection when necessary to relieve respiratory obstruction. There would appear to be little justification for resecting the pulmonary lesions, for they are usually multiple and bilateral, and probably only very slowly progressive.

Authenticity of Recorded CASEs.-The number of amyloid tumours of the entire respiratory tract recorded up to 1935 was 95 cases (Kramer and Som), since when many more have been described. These cases include many in which the amyloid staining reactions had been omitted or had been negative, and in the latter instances the lesions have often been described as characteristic of pre-amyloid or para-amyloid.

The majority of cases have been amyloid tumours of the larynx or vocal cords, which, according to Ash and Schwartz (1944), and Kelly and Craik (1952), are often misnamed and would be better described as hyaline nodes. In 1948 Stark and McDonald re-examined the respiratory tract amyloid tumours in the surgical files of the Mayo Clinic. Adequate material for further study was available in 24 out of 28 cases, and by carefully controlled use of amyloid stains they were able to confirm the diagnosis in 15 cases. They considered that characteristic pathological features were: (1) Homogeneous amyloid material in flakes and concentric layered masses; (2) characteristic staining of the material with one or more of the amyloid stains.

If the recorded cases of lower respiratory tract amyloid tumours are examined critically, using similar standards as far as it is applicable, it is seen that only two cases are not acceptable. In Hallermann's first case the naked-eye appearance was typical, as was the histological distribution of the "amyloid," but staining reactions with iodine and methyl violet were negative. In the case recorded by Weismann and associates the "amyloid" was on the outside of the bronchial walls and around the pulmonary vessels. There were no sub-epithelial deposits, no accumulations around mucous glands, and staining reactions showed a lack of uniformity. It is probable that this case is the one which was mentioned by Stark and McDonald as being not amyloid but " an inflammatory tumefaction of the peribronchial lymph nodes with involvement of the bronchi."

\section{COMMENT}

It is surprising that in an era of thoracic exploration amyloid tumours of the lower respiratory tract have received so little attention, and that in most of the recorded cases the diagnosis has only been made after post-mortem examination.

Not very long ago bronchial adenomas were frequently diagnosed as carcinomas, largely through an unawareness of the existence of adenomas. It is possible that for similar reasons many amyloid tumours are misdiagnosed as fibrosis. This in itself may not be of great importance to the patients, but histological reports of "fibrosis" leave uncertainty in the minds of clinicians, who are familiar with fibrosis associated with bronchial neoplasms, tuberculosis, and nonspecific inflammation.

Descriptions of the bronchial lesions in the recorded cases of amyloid tumours are remarkably uniform, and it is certain that a correct diagnosis could usually be made from the bronchoscopic appearances, provided that the condition is kept in mind.

\section{SUMMARY}

A 48-year-old man was investigated because of a recent haemoptysis. On bronchoscopy a peculiar polypoid thickening of the mucosa of the lower trachea and main bronchi and an isolated tumour in the right lower lobe bronchus were seen. Histological examination of tissues removed from the 
bronchi showed lesions which are characteristic of amyloid deposits of the respiratory tract. No generalized amyloidosis was present.

The 10 previously recorded cases of amyloid tumours of the lower respiratory tract are briefly reviewed, and their clinical and pathological features are discussed.

It is considered that the bronchoscopic and histological appearances are sufficiently distinctive for a correct diagnosis to be made in the majority of cases, except when the lesions affect only the parenchyma of the lungs.

I wish to express my thanks to Dr. A. C. C. Hughes and $\mathrm{Mr}$. Leslie Temple for making available to me their clinical records of this patient.

\section{REFERENCES}

Ash, J. E, and Schwartz, L. (1944). Trans Amer Acad. Ophthal. Otolaryng., 48, 323. Quoted by Kelly and Craik. Balser, W. (1883). Virchows Arch. path. Anat., 91, 67. Beckert, G. (1917). Frankfurt. Z. Path., 20, 1.

Falconer, B. (1938). Acta oto-laryng., Stockh., 26, 353. Figi, F. A. (1942). Proc. Mayo Clin., 17, 239.

Glockner, A. (1900). Virchows. Arch. path. Anat., 160, 583

Hallermann, W. (1928). Frankfurt. Z. Path., 36, 471.

Haynes, A. L., Clagett, O. T., and McDonald, J. R. (1948). Surger 24,120 .

Herxheimer, G. (1903). Virchows Arch. path. Anat., 174, 130.

Kelly, H. D. Brown, and Craik, J. E. (1952). J. Laryng., 66, 339. Kramer, R., and Som, M. L. (1935). Arch. Otolaryng., Chicago, 21, 324

Meyer, O. (1911). Frankfurt. Z. Path., 8, 304.

New, G. B., and Erich, J. B. (1938). Arch. Otolaryng., Chicago, 28 841

Pollak, E. (1914). Z. Laryng. Rhinol., 7, 25. Quoted by Kramer and Som.

Stark, D. B., and McDonald, J. R. (1948). Amer. J. clin. Path., 18, 778

Weismann, R. E., Clagett, O. T., and McDonald, J. R. (1947). J. thorac. Surg., 16, 269.

Werdt, F. von (1908). Beitr. path. Anat., 43, 239. 